\title{
Gestão do cuidado e da clínica no atendimento aos usuários da Estratégia Saúde da Família
}

\section{Clinical and Care management in the assistance of users of the Family Health Strategy Gestión del cuidado y de la clínica en el atendimiento a los usuarios de la Estrategia Salud de la Familia}

\section{Recebido: 06/04/2018 \\ Aprovado: 20/11/2018 \\ Publicado: 29/01/2019}

\section{Antonio Germane Alves Pinto ${ }^{1}$ Maria Dayanne Luna Lucetti ${ }^{2}$ Kelly Fernanda Silva Santana ${ }^{3}$ Adriana de Moraes Bezerra ${ }^{4}$ Maria Corina do Amaral Viana ${ }^{5}$ Maria Salete Bessa Jorge 6}

O objetivo do estudo foi analisar a prática clínica e a gestão do cuidado no cotidiano da Estratégia Saúde da Família com ênfase nos limites, potencialidades e desafios para a consolidação do Sistema Único de Saúde. Trata-se de estudo de natureza qualitativa, realizado com 42 usuários e 31 trabalhadores de saúde do Município de Fortaleza, Ceará. Os resultados evidenciam que as ações de saúde no território são regidas pela eficiência produtiva da equipe de saúde. Emergiram como categorias: os limites e tensões no atendimento, as potencialidades cotidianas, e os desafios para gestão do cuidado e da clínica. As limitações estruturais e operacionais para o cuidado em saúde são superadas pelas relações intersubjetivas entre a equipe e a comunidade. A gestão do cuidado se verticaliza nos processos de trabalho e se demonstra frágil no que diz respeito ao alcance de condições de saúde adequadas. Considera-se que a prática clínica e o cuidado em saúde tangenciam as diretrizes político-institucionais do Sistema Único de Saúde e o direito à saúde ainda é parcialmente garantido no cotidiano assistencial.

Descritores: Guia de Prática Clínica; Estratégia Saúde da Família; Atenção Primária à Saúde; Integralidade em saúde; Sistema Único de Saúde.

The aim of this study was to analyze the clinical practice and management of care in the daily life of the Family Health Strategy with emphasis on limits, potentials, and challenges for the consolidation of the Unified Health System. It is a qualitative study, carried out with 42 users and 31 health workers of the Municipality of Fortaleza, Ceará. The results show that the health actions in the territory are governed by the productive efficiency of the health care team. The following categories emerged: limits and tensions in the assistance, daily potential, and challenges for care and clinical management. The structural and operational limitations for health care are overcome by the inter-subjective relations between the team and community. The management of care is vertical in the work processes and demonstrates frailties in reaching an adequate health conditions. It is considered that the clinical practices and health care touch on the policies and institutional guidelines of the Unified Health System and the legal right to health is still partially guaranteed in daily care.

Descriptors: Practice Guideline; Family Health Strategy; Primary Health Care; Integrality in Health; Unified Health System.

El objetivo del estudio fue analizar la práctica clínica y la gestión del cuidado en el cotidiano de la Estrategia Salud de la Familia con énfasis en los límites, potencialidades y desafíos para la consolidación del Sistema Único de Salud. Se trata de un estudio de naturaleza cualitativa, realizado con 42 usuarios y 31 trabajadores de salud del municipio de Fortaleza, Ceará. Los resultados demuestran que las acciones de salud en el territorio son regidas por la eficiencia productiva del equipo de salud. Emergieron como categorías, los límites y tensiones en el atendimiento, las potencialidades cotidianas y los desafíos para la gestión del cuidado y de la clínica. Las limitaciones estructurales y operacionales para el cuidado en salud son superadas por las relaciones intersubjetivas entre el equipo y la comunidad. La gestión del cuidado se verticaliza en los procesos de trabajo y se demuestra frágil al alcance de condiciones de salud adecuadas. Se considera que la práctica clínica y el cuidado en salud se valen de las directrices político-institucionales del Sistema Único de Salud y el derecho a la salud aún es parcialmente garantizado en el cotidiano asistencial.

Descriptores: Guía de Práctica Clínica; Estrategia de Salud Familiar; Atención Primaria de Salud; Integralidad en salud; Sistema Único de Salud.

1. Enfermeiro. Especialista em Saúde da Família. Mestre em Cuidados Clínicos em Saúde. Doutor em Saúde Coletiva. Docente do Mestrado Profissional em Saúde da Família da Rede Nordeste de Formação em Saúde da Família. Professor do Departamento de Enfermagem da Universidade Regional do Cariri (URCA), CE, Brasil. ORCID: 0000-0002-4897-1178 E-mail: germanepinto@hotmail.com

2. Enfermeira. Especialista em Estratégia Saúde da Família. Especialista em Educação na Saúde para Preceptores do SUS. Mestre em Enfermagem. Professora do Departamento de Enfermagem da URCA, Cariri, CE, Brasil. ORCID: 0000-0002-8664-4901E-mail: dayanne.cmae@gmail.com

3. Enfermeira. Especialista em Enfermagem do Trabalho. Mestre em Enfermagem. Professora Substituta do curso de Enfermagem da URCA, Cariri, CE, Brasil. ORCID: 0000-0002-7254-1944 E-mail: kellyfernandassantana@hotmail.com

4. Enfermeira. Especialista em Gerontologia. Mestre em Enfermagem. Professora do Departamento de Enfermagem da URCA, Cariri, CE, Brasil. ORCID: 0000-0003-0929-4685 E-mail: adriana1mb@hotmail.com

5. Enfermeira. Doutora em Enfermagem. Professora do Departamento de Enfermagem e do Mestrado Profissional em Saúde da Família da Rede Nordeste de Formação em Saúde da Família (Nucleadora). Mestrado Profissional em Saúde da Criança e Adolescente. Mestrado Acadêmico em Enfermagem, URCA, Cariri, CE, Brasil. ORCID:0000-0002-6890-9400 E-mail: corina.viana@urca.br

6. Enfermeira. Mestre em Enfermagem Psiquiátrica. Doutora em Enfermagem. Pós-Doutora em Saúde Coletiva. Professora Titular do Programa de Pós-Graduação em Saúde Coletiva, da Universidade Estadual do Ceará, Fortaleza, CE, Brasil. ORCID: 0000-0001-6461-3015 E-mail: masabejo@uece.br

*Fonte de Financiamento: Bolsa de Doutorado - Fundação Cearense de Apoio ao Desenvolvimento Científico e Tecnológico - FUNCAP. 


\section{INTRODUÇÃO}

$\mathbf{P}$ ara efetivar universalidade,

os princípios integralidade equidade no Sistema Único de Saúde (SUS), deve-se dispor de uma rede assistencial que priorize a Atenção Primária à Saúde (APS) e mantenha a resolutividade pela integração dos serviços entre seus níveis de complexidade. Nesse processo, a Estratégia Saúde da Família (ESF) se torna relevante pelo seu perfil assistencial mais sensível às demandas e necessidades de saúde da população ${ }^{1}$.

A APS opera modificações assistenciais com impacto significativo nos indicadores de saúde e assistência ${ }^{2}$. As políticas públicas de incentivo à ampliação da cobertura da ESF nos municípios são mecanismos institucionais que estabelecem a execução de práticas voltadas para promoção da saúde e prevenção de doenças. Entretanto, são os processos de trabalho das equipes, a integralização dos fluxos de atendimento e a plenitude participativa que estão diretamente relacionados com 0 reconhecimento da cidadania e a saúde da população ${ }^{3}$.

Nesse sentido, para que a ESF legitime seu caráter estratégico de inclusão de segmentos populacionais nos serviços de saúde, deverá respaldar este processo de trabalho na realidade cotidiana. A política de saúde se implementa na realidade da atenção à saúde por um conjunto de condições: maior disponibilidade de recursos financeiros para o setor saúde, alocação de recursos entre esferas de governo, e organização local da rede de serviços de saúde para garantia do acesso universal, integral e equânime dos usuários ${ }^{4}$.

Nos grandes e médios centros urbanos se tornam relevantes as tensões nas portas de entrada dos serviços de saúde. Primordialmente, o entendimento sobre a demanda apresentada nas unidades de saúde e suas causalidades indica a possibilidade de inovação das práticas de gestão pública, políticas sociais condizentes com as necessidades da população e, também, a diminuição das iniquidades prevalentes no território.
Cotidianamente, urgem modificações na forma dos profissionais de saúde lidarem com as pessoas que necessitam do atendimento. A contribuição maciça da lógica assistencial prescritiva parte dos próprios profissionais de saúde e das requisições dos usuários. Determinados pelas condições tensas de atendimento, formatos clínicos pautados na cura fragilizam o cuidado em situações agudas e/ou crônicas de adoecimento. A consistência entre o cuidado e a clínica exige a busca por práticas de promoção da saúde e empoderamento dos usuários.

Nesse contexto, este estudo tem como objetivo analisar a prática clínica e a gestão do cuidado no cotidiano da Estratégia Saúde da Família com ênfase nos limites, potencialidades e desafios para a consolidação do Sistema Único de Saúde.

\section{MÉTODO}

Estudo de natureza qualitativa com enfoque hermenêutico, pautado na interpretação crítica e reflexiva do cotidiano ${ }^{5}$. A pesquisa foi realizada no Município de Fortaleza, capital do Estado do Ceará, Nordeste Brasileiro. Em 2013, a população coberta pelo SUS era de 5.733.859 habitantes de um total estimado de 8.530.155 habitantes no Estado. A cobertura pela ESF no âmbito estadual era de aproximadamente 67,22\%, sendo que a capital mantinha apenas $33,15 \%{ }^{6}$.

Os participantes do estudo foram 31 profissionais de saúde de duas equipes da ESF e 42 usuários cadastrados e atendidos. A seleção dos participantes ocorreu na aproximação ao campo de estudo, com seleção prévia das equipes junto ao núcleo gestor municipal. A composição amostral se delimitou pela relação das equipes com os usuários e pela discussão ampliada e significativa sobre o objeto deste estudo.

Foram utilizadas as técnicas de coleta de dados: entrevista semiestruturada, grupo focal e observação sistemática. A aplicação dos instrumentos foi realizada no período de abril de 2011 a janeiro de 2012.

Após autorização da pesquisa, foram realizadas entrevistas com roteiro de pauta 
sobre o tema do estudo. Os grupos focais foram realizados com a participação múltipla, por usuários e pela equipe de saúde. Os grupos tiveram entre seis e oito participantes, com agendamento prévio e tempo médio de duração das sessões de 45 minutos. A observação sistemática foi pautada nas questões sobre resolutividade, ações para promoção da saúde, e funcionamento da unidade de saúde.

A análise dos dados foi pautada pelo processo compreensivo e interpretativo fundamentado pela hermenêutica crítica ${ }^{5}$. Foram apresentadas convergências e divergências, em complementaridades e diferenças ilustradas com discursos dos participantes, observações, e discutidas à luz da literatura pertinente. A divisão descritiva por áreas de adscrição das equipes foi suprimida para evitar o reconhecimento dos participantes.

A pesquisa foi aprovada pelo Comitê de Ética em Pesquisa, Protocolo № 10724452-7 conforme Resolução 466/12 do Conselho Nacional de Saúde?

\section{RESULTADOS}

Participaram 31 profissionais de saúde de duas equipes da ESF e 42 usuários cadastrados e por elas atendidos.

No processo de interpretação foram analisadas narrativas e observações que entrecruzam a situação das diretrizes para consolidação do SUS e o modo de assistência à população pela ESF. Dispõem-se três categorias temáticas emergentes do cotidiano, relacionadas aos: Limites e tensões do cotidiano, Possibilidades cotidianas e, Desafios para a gestão do cuidado e da clínica.

\section{Limites e tensões no atendimento}

A operacionalidade cotidiana das ações da ESF no território é movida pela eficiência produtiva da equipe de saúde. Convergem práticas de cuidado voltadas para o atendimento ambulatorial da demanda recorrente:

A máquina não para. Todo dia tem gente para atender. E não é coisa pouca não. É gente precisando da gente. Se a gente parar muito para conversar, vai ficar gente sem ser atendido. Então, o que importa mesmo no dia-adia é o que a gente faz. Se é promoção, se é integralidade, fica para depois. O que importa é atender bem. E com qualidade. (Médico)

O difícil aqui é conseguir uma ficha para ser atendido. Eu chego cedo. Para ser atendido cedo. Às vezes, o médico atrasa. Mas ele deve ter os motivos dele. Mas quando chega, vai logo atendendo. Faz o que tem para fazer. Isso é bom. Eu não tenho o que reclamar. (Usuário)

Complementa-se no território, situações em que usuários em processo de adoecimento e na busca pela manutenção da saúde enfrentam a insuficiente cobertura assistencial em relação à extensa dimensão geográfica e demográfica do espaço urbano. Concomitantemente, condições de risco e vulnerabilidades prevalentes se agregam à necessidade de atenção à saúde universal e equânime, entre equipe de saúde e usuários: o posto é muito longe. Eu sou doente. Para andar até lá, vou ficar mais doente ainda. O meu filho quando está de folga me leva de carro. É muito longe. Acho que já estava na hora de ter um posto mais perto aqui da gente. (Usuário, Idoso)

Fica difícil a gente dar um atendimento bem efetivo porque a área é muito grande e populosa. É muita gente e o pior, a área também é grande. $O$ povo dá um jeito $e$ procura as ajudas, carona, companhia, mas mesmo assim fica complicado vir ao posto pela distância. (Agente Comunitária de Saúde)

Enfatiza-se que a APS em saúde é responsável pela elucidação da maioria dos casos clínicos entre os usuários de um território. Caso contrário, o agir clínico na superficialidade do atendimento fragiliza o cuidado e, ao longo do tempo, vai remetendo a população às condições de adoecimento, ou seja, a assistência torna-se limitante para resolutividade terapêutica:

Está difícil demais manter a saúde. Para gente conseguir alguma melhora, tem que ter um conhecido. No posto é gente demais. Hoje em dia, se não tiver uma pessoa que ajude, fica difícil. (Usuário)

Meu caso é muito complicado. Se eu não cuidar, eu vou morrer. Porque nesse mundo, é cada um por si. A dificuldade que a gente encontra nesse SUS é a falta de atenção. A pessoa lhe atende e não olha nem você direito. Meu problema é grave! [...] É gente demais, não dá tempo nem o doutor olhar pra gente. (Usuários)

Em observações, agregam-se dificuldades da equipe da ESF em possibilitar resolução dos problemas e situações de adoecimentos e agravos na lida ambulatorial. Convergem situações de demora excessiva, baixa comunicação em rede e descontinuidade do cuidado: 
Neurologista, ortopedista, neuropediatra, otorrino, gastroenterologista são uns encaminhamentos que dificilmente a gente vai conseguir. Pequenas e grandes cirurgias também. Porque não tem vaga no sistema. Eu não sei por que o sistema é precário, entendeu? Essa central de marcação de consulta eu acho que deveria fazer uma reforma urgentemente, porque pacientes vão morrer se não mudar. (Agente Comunitário de Saúde) É preciso trabalhar em equipe, em rede. Se encaminho um paciente para outro serviço é porque não tenho condições de resolver aquela situação. O outro serviço precisa conhecer a nossa realidade para entender porque encaminhei e dar continuidade ao tratamento. Muitas vezes, isso não acontece e o prejudicado com essa fragmentação é, principalmente, o paciente. O SUS ainda não trabalha em rede. (Médico)

Em situações de urgência e complicação clínica, as narrativas dos usuários no grupo focal, bem como nos discursos de trabalhadores de saúde, evidenciam o caminho do hospital como lugar de atendimento imediato:

[...] quando acontece algo mais sério, a gente corre para o hospital. [...] Nenhum dos dois presta. O hospital pelo menos funciona direto. [...] Demora, mas a gente sabe que vai ser atendido. No Posto de saúde é como um "escape". A gente tenta para ver se dá certo. [...] Se o seu filho ou uma pessoa sua passasse mal, o que o senhor ia fazer? Tudo que for para ajudar é bom. Eu pago é um carro para ir ser atendido no [hospital], mas não deixo um parente meu morrer à míngua. (Usuários)

Eles vão muito para o hospital nos casos de urgência e emergência. Tem gente que leva as crianças com febre, agora com a Dengue, os adultos mais graves. E, principalmente, as pessoas com pressão alta. (Agente Comunitária de Saúde)

O hospital é muito importante para nosso trabalho aqui no posto. Infelizmente, não existe uma comunicação mais forte entre a gente. Nós encaminhamos os casos mais urgentes, mas não temos retorno contínuo. E ainda tem o pessoal da comunidade que vai direto à emergência sem nem passar no posto. (Médico)

\section{Potencialidades cotidianas}

De modo divergente das situações analisadas, as insuficiências estruturais e operacionais para o cuidado em saúde são superadas com iniciativas operadas pela equipe da ESF. Destaca-se a busca pela resolutividade assistencial potencializada pela interação entre quem precisa e a ação que se faz para atenuar a dor e/ou sofrimento da população: $O$ PSF, do jeito que está não consegue ser porta de entrada, pela demanda e pelas condições de atendimento. A única coisa que a gente tem de bom no trabalho é a nossa integração em equipe. Aqui todo mundo tenta ajudar um ao outro. (Médico)
A gente tenta procurar marcar exames que são mais urgentes - dar outras soluções - correr atrás para resolver. (Enfermeira)

Eu faço o que posso, na dificuldade, mas mantenho a esterilização do material e dou um jeito no restante para não deixar o usuário sem atendimento. (Dentista) Sempre que tem algo mais complicado - a gente faz um mutirão de ajuda - é uns ajudando os outros. É assim. (Agentes Comunitários de Saúde)

Converge nas relações empreendidas pela equipe da ESF a operação de uma prática cuidadora mais próxima dos sujeitos. A ampliação relacional, o compartilhamento de saberes nas abordagens e técnicas utilizadas, tornam o atendimento singularizado. Assim, é possível a obtenção de resultados positivos pela corresponsabilização mútua:

[...] quando a gente consegue ser atendido, eles dão muita atenção. $O$ doutor daqui do posto já chegou até ir lá em casa pra saber se meus exames tinham dado certo, se minha cirurgia tinha ficado boa. [...] se não fosse esses anjos (ACS). A nossa saúde estava ruim. Mas eles fazem de tudo que podem. Se não fosse esse daqui (ACS), não tinha conseguido nunca fazer meu tratamento direito. (Usuários)

Sempre que a gente pode, a gente tenta dar uma atenção maior aos casos mais graves, as gestantes, os hipertensos mais complicados, as crianças menores. $O$ número de marcação de consultas e exames é pequeno $e$ a gente tem que priorizar, quando pode. (Enfermeira) As coisas só funcionam quando as equipes dão tudo de si para fazer bem aquilo que precisa. E também os pacientes cooperam e buscam se cuidar. Quem faz as coisas acontecer aqui são as pessoas que se comprometem em fazer direito. (Médico)

Complementa-se à dinâmica de trabalho em áreas de risco e vulnerabilidade, a ampliação subjetiva contextualizada pela situação social dos usuários durante 0 trabalho da ESF. Os protocolos terapêuticos, embora ainda sejam roteirizados pelos programas prioritários, são adaptados ou subjazidos pelas condições de vida da população:

Todo mês a gente checa se eles estão sendo acompanhados, se fizeram os exames e se estão tomando o remédio direito. É tudo controlado pela gente da equipe. (Enfermeira)

Acrescenta-se que a desassistência extrapola a atenção à saúde e perpassa pela deficiente oferta de água potável, esgoto sanitário, coleta de lixo, alimentação, entre outros. (Observação)

É preciso dar um jeito de orientar a população a partir do que ela tem. (Médico)

Em sentido resolutivo, a programação regular de atividades preventivas no trabalho 
da ESF evita situações problemáticas na vida dos usuários:

Com essa consulta mensal, a gente evita atraso nas vacinas, problemas comuns nessa idade $e$, principalmente, que elas fiquem doentes com frequência. (Enfermeira, ao atender uma criança e mãe no dia agendado para a puericultura na ESF)

Emerge uma confluência de sentidos assistenciais na ESF que envolvem ações prescritivas e controle de riscos e agravos. A promoção da saúde resgata espaços entre as condutas superficiais e distantes do sentido mais amplo da saúde. A rede de atenção básica mantém seu perfil assistencial efetivando atividades preventivas, em destaque, mecanismos de grupo, acompanhamento domiciliar e educação em saúde:

As atividades de promoção são basicamente as palestras $e$ os grupos que a gente faz com as gestantes, principalmente. Sempre tem alguma atividade na sala de espera, a gente orienta antes das consultas. (Enfermeira)

Tem o pessoal das endemias que ajuda muito na prevenção das doenças. Sempre que tem campanha da dengue, eles ajudam muito na parte de orientação da comunidade, visitando com a gente (Agente Comunitária de Saúde).

A gente sabe que é preciso se cuidar antes que a doença venha. Comer direito, evitar sal e gordura. Tudo isso a gente escuta aqui no posto. Eles orientam direitinho (Usuário).

\section{Desafios para gestão do cuidado e da clínica}

0 processo de gestão das unidades de saúde ainda se apresenta verticalizado nas questões operacionais relacionadas à demanda de cuidado em saúde do território. A circularidade do poder de decisão e de governabilidade se encerra quando são apresentados problemas envolvendo níveis mais ascendentes da gestão, como se expressa:

o problema é que vivemos um período de falta de credibilidade no sistema. 0 profissional perdeu o crédito no SUS devido às tentativas frustradas de transformação. Com isso, ele prefere não tentar mais e fazer do jeito que dá certo. Por um lado, a prática clínica do dia-a-dia é que resolve mesmo os problemas da população, mas, para isso, tem que incentivar o paciente a buscar seus direitos, reivindicar seu tratamento correto, etc. Por outro, nem sempre é isso que o nível político e gerencial do SUS deseja. É mais fácil para a gestão ter um profissional que dá qualquer jeito e não faz com que o paciente tenha consciência de suas necessidades. (Médico)
É preciso melhorar o atendimento da unidade. Nós temos a famosa grande roda mensal, que é com a coordenadora do posto, certo. Todos os meses. Eu participo todo mês, mas tem colega que não vai mais porque não vê o negócio andar. [...] A grande roda, nós nos reunimos, muitos assuntos são debatidos, mas nada! É dito, é falado, mas resposta que é bom nada. (Agentes Comunitários de Saúde)

Observa-se entre os usuários, a disposição expressiva e quase sempre desesperada de pessoas que vêm às unidades de saúde no limite da necessidade e com poucas opções de resolução dos seus problemas ou processos de adoecimento. 0 controle gerencial é insuficiente, segundo os relatos da equipe:

A organização é aquela que você [pesquisador] viu naquele dia. $O$ pessoal tudo misturado. Tem vezes que é pior. Tem vezes que tem até briga. (Agente Comunitária de Saúde)

Todo dia é gente que aparece reclamando. É muita doença e pouco doutor para atender (Médico)

Essas pessoas procuram muito o atendimento médico e muitos, desse pessoal, é de fila de espera que ficam muito tempo esperando sua vez. Mas a gente só tem autorização para distribuir 25 senhas. (Assistente Administrativo - Recepção)

Eu fico aqui direto, tentando fazer o melhor, conversando e checando se toda a equipe está atendendo. (Coordenadora da Unidade)

\section{DISCUSSÃO}

A assistência prestada pelas equipes da ESF ainda prioriza ações voltadas para a produção de procedimentos e cuidados imediatos. 0 acesso aos serviços de saúde se processa socialmente nas relações de necessidade, demanda e oferta. Entretanto, é preciso estabelecer um processo reflexivo da prática assistencial para reconhecimento de condições sociais, determinantes de saúde e cidadania ${ }^{8}$.

Na organização da ESF, a localização das Unidades de Saúde é também estratégica para a acessibilidade da população. A programação de ações e serviços com resolutividade e a promoção da saúde comunitária exigem uma distribuição espacial adequada às condições de vida dos usuários e com proximidade dos seus contextos de moradia. Em detrimento da universalidade, $\mathrm{o}$ acesso restrito $\mathrm{e}$ desproporcional aos serviços de saúde no SUS, se torna um entrave para a consolidação da atenção à saúde ${ }^{3,8,9}$. 
Os atendimentos clínicos que exigem atenção especializada esbarram na insuficiente logística de regulação dos encaminhamentos ${ }^{10}$. A ESF incorpora em sua dinâmica assistencial, a programação de atividades e intervenções em rede nos seus diversos níveis de complexidade.

A longitudinalidade do cuidado se torna efetiva com o acompanhamento do usuário pela equipe de saúde, ao longo do tempo. $\mathrm{Na}$ APS, a continuidade assistencial é considerada característica central na garantia do acesso integral aos procedimentos, serviços e atendimentos especializados necessários para o alcance da resolutividade no tratamento ${ }^{11,12}$.

Historicamente, a centralidade hospitalar é hegemônica na operacionalidade assistencial brasileira. Entretanto, as políticas almejam o incentivo para ampliação da ESF em diferentes ações diretas e transversais (educação em saúde, Núcleos de Apoio a Saúde da Família, gestão participativa e humanização). Enfatiza-se que a inversão da lógica hospitalocêntrica requisita qualificação da atenção básica, resolutividade clínica e integração efetiva com a atenção especializada e hospitalar ${ }^{13,14}$.

As relações entre equipe de saúde e usuários afetam as pessoas envolvidas no sentido de estimular a solidariedade e a motivação no cuidado em saúde. Cônscios da responsabilidade sanitária, as equipes que reconhecem os problemas operacionais e ativam movimentos para resolução dos problemas promovem a transformação do processo de trabalho ${ }^{15}$.

A consolidação da atenção à saúde se efetiva nas ações da prática assistencial e nas relações entre as pessoas envolvidas com o cuidado em saúde. A resolutividade da clínica se constrói pelas condições ofertadas ao trabalho assistencial e na disposição planejada de insumos, medicamentos e procedimentos. Desse modo, é possível vivenciar um cotidiano transversalizado por práticas cuidadoras integrais, regulares e resolutivas ${ }^{16,17}$.

$\mathrm{Na}$ busca por resolutividade, a ESF estabelece prioridades para o acompanhamento, controle e monitoramento das situações de saúde da população. Entretanto, as ações cotidianas devem reconhecer os determinantes sociais da população também como horizonte para a eficácia de suas intervenções ${ }^{18}$.

$\mathrm{Na}$ territorialidade do adoecimento, considerar as condições de vida da população e efetivar modos diversos no cuidar é garantir equânimes possibilidades para manutenção do processo saúde-doença ${ }^{18-20}$.

Quando se propõe a gestão compartilhada, fica pressuposta a participação ativa dos envolvidos e o reconhecimento da subjetividade cidadã. 0 poder se dissolve e, ao mesmo tempo, se fortalece pela elucidação participativa dos sujeitos no diálogo em torno de um problema comum. Se o processo difere em parte ou no todo, resultará em método assíncrono da realidade a ser transformada ${ }^{21}$.

As demandas apresentadas nas salas de espera e nas recepções são apenas as "pontas do iceberg", que indicam uma base dos determinantes de uma saúde desequilibrada em relação à manutenção da vida saudável. Atuar na superficialidade da demanda é atingir um nível semi-básico e quase recorrente dos problemas do território ${ }^{22,23}$. A organização do atendimento é um desafio contínuo para consolidar a ESF como ordenadora da rede de cuidados.

As situações limítrofes entre a oferta e a demanda na ESF precisam conciliar a redução dos déficits assistenciais por um lado, e incidir novos trajetos para mobilizar o território ao processo de vida saudável, por outro. Eis o risco contínuo do recrudescimento da avaliação sobre as práticas de saúde tidas como resolutivas apenas no fechamento de processos mórbidos de adoecimento agudo ou crônico, em torno da eficiência assistencial.

\section{CONCLUSÃO}

O presente estudo limita-se pela focalização territorial exigida pelo processo de aprofundamento interpretativo das relações cotidianas da ESF. Entretanto, os arranjos temáticos discutidos expõem situações vivenciadas por equipes de saúde da família em diferentes contextos. 
O envolvimento analítico, crítico e reflexivo amplia as dimensões e olhares sobre o objeto de estudo, dispondo evidências subjetivas para a gestão da clínica e do cuidado no território vivo, singular e diverso.

Numa síntese analisadora, a ESF ainda apresenta um espaço distante entre a pretensão da política institucional do SUS e a realidade cotidiana do cuidado e da prática clínica.

Contrapõem-se incentivos para implantação, ampliação e custeio para as atividades da ESF, e um agir clínico ainda fragmentado em seus fluxos assistenciais. Desse modo, exprime-se uma limitação da atenção à saúde pela insuficiente universalidade, bem como pela fragmentação do cuidado e iniquidades sociais recorrentes.

Pela possibilidade de tornar estratégica a implementação da APS em saúde no sentido de inverter a lógica hospitalocêntrica e amplificar o cuidado territorial, o planejamento deve respaldar a participação dos sujeitos com suas necessidades e demandas.

As relações intersubjetivas entre trabalhadores de saúde e usuários devem compor o planejamento comunitário e participativo das ações de saúde. A eficácia da atenção à saúde se remete às soluções reais da convivência urbana e do acesso integral aos cuidados de saúde.

\section{REFERÊNCIAS}

1. Fertonani HP, Pires DEP, Biff D, Scherer MDA. Modelo assistencial em saúde: conceitos e desafios para a atenção básica brasileira. Ciênc Saúde Colet. [Internet]. 2015 [citado em 15 jun 2017]; 20(6):1869-78. DOI: http://dx.doi.org/10.1590/1413-

81232015206.13272014

2. Boing AF, Vicenzi RB, Magajewski F, Boing AC, Moretti-Pires RO, Peres KG, et al. Redução das internações por condições sensíveis à atenção primária no Brasil entre 1998-2009. Rev Saúde Pública [Internet]. 2012 [citado em 15 jun 2017]; 46(2):359-66. DOI: http://dx.doi.org/10.1590/S003489102012005000011
3. Silva LA, Casotti CA, Chaves SCL. A produção científica brasileira sobre a Estratégia Saúde da Família e a mudança no modelo de atenção. Ciênc Saúde Colet. [Internet]. 2013 [citado em 18 jun 2017]; 18(1):221-32. DOI: http://dx.doi.org/10.1590/S1413-

81232013000100023

4. Sousa MF, Hamann EM. Saúde da família no Brasil: estratégia de superação da desigualdade na saúde? Physis [Internet]. 2009 [citado em 18 jun 2017]; 19(3):711-29. DOI: $\quad$ http://dx.doi.org/10.1590/S010373312009000300009

5. Minayo MCS. O desafio do conhecimento: pesquisa qualitativa em saúde. 14ed. São Paulo: Hucitec; 2014.

6. Ministério da Saúde (Br), Secretaria de Atenção à Saúde, Departamento da Atenção Básica. Evolução do credenciamento e implantação da estratégia Saúde da Família. Dados do Estado do Ceará e capital [Internet]. Brasília, DF: Ministério da Saúde; 2013 [citado em 18 jun 2017]; Disponível em:

http://dab.saude.gov.br/historico_cobertura_ sf/historico_cobertura_sf_relatorio.php

7. Conselho Nacional de Saúde (Brasil). Resolução № 466 de 12 de dezembro de 2012 [Internet]. Aprova as diretrizes e normas regulamentadoras de pesquisas envolvendo seres humanos [Internet]. Brasília, DF: CNS; 2012 [citado em 18 jun 2017]. Disponível em: http://bvsms.saude.gov.br/bvs/saudelegis/c ns/2013/res0466_12_12_2012.html

8. Jesus WLA, Assis MMA. Revisão sistemática sobre o conceito de acesso nos serviços de saúde: contribuições do planejamento. Ciênc Saúde Colet. [Internet]. 2010 [citado em 18 jun 2017]; 15(1):161-70. Disponível em: http://www.scielo.br/pdf/csc/v15n1/a22v1 5n1.pdf

DOI: http://dx.doi.org/10.1590/S1413-

81232010000100022

9. Pessoa VM, Rigotto RM, Carneiro FF, Teixeira ACA. Sentidos e métodos de territorialização na atenção primária à saúde. Ciênc Saúde Colet. [Internet]. 2013 [citado em 18 jun 2017]; 18(8):2253-62. 
DOI:http://dx.doi.org/10.1590/S141381232013000800009

10. Almeida PF, Giovanella L, Nunan BA. Coordenação dos cuidados em saúde pela atenção primária à saúde e suas implicações para a satisfação dos usuários. Saúde Debate [Internet]. 2012 [citado em 18 jun 2017]; 36(94):375-91.

http://dx.doi.org/10.1590/S0103-

11042012000300010

11. Cunha EM, Giovanella L. Longitudinalidade/continuidade do cuidado: identificando dimensões e variáveis para a avaliação da Atenção Primária no contexto do sistema público de saúde brasileiro. Ciênc Saúde Colet. [Internet]. 2011 [citado em 18 jun 2017]; 16(Suppl1):1029-42. Disponível em:

http://www.scielo.br/pdf/csc/v16s1/a36v1 6s1.pdf

12. Arce VAR, Sousa MF. Práticas de longitudinalidade no âmbito da Estratégia Saúde da Família no Distrito Federal. Cad Saúde Colet. [Internet]. 2014 [citado em 18 jun 2017]; 22(1):62-8.

DOI:http://dx.doi.org/10.1590/1414462X201400010010

13. Cecilio LCO, Andreazza R, Carapinheiro G, Araújo EC, Oliveira LA, Andrade MGG, et al. A Atenção Básica à Saúde e a construção das redes temáticas de saúde: qual pode ser o seu papel? Ciênc Saúde Colet. [Internet]. 2012 [citado em 18 jun 2017]; 17(11):2893-902. DOI: http://dx.doi.org/10.1590/S141381232012001100006

14. Reis RS, Coimbra LC, Silva AAM, Santos AM, Alves MTSSB, Lamy ZC, et al. Acesso e utilização dos serviços na Estratégia Saúde da Família na perspectiva dos gestores, profissionais e usuários. Ciênc Saúde Colet. [Internet]. 2013 [citado em 18 jun 2017]; 18(11):3321-31.

DOI:

http://dx.doi.org/10.1590/S1413-

81232013001100022

15. Norman AH, Tesser CD. Acesso ao cuidado na Estratégia Saúde da Família: equilíbrio entre demanda espontânea e prevenção/promoção da saúde. Saúde Soc. [Internet]. 2015 [citado em 18 jun 2017]; 24(1):165-79.

DOI:
http://dx.doi.org/10.1590/S010412902015000100013

16. Castro ALB, Machado CV. A política federal de atenção básica à saúde no Brasil nos anos 2000. Physis [Internet]. 2012 [citado em 18 jun 2017]; 22(2):477-506. DOI: http://dx.doi.org/10.1590/S0103-

73312012000200005

17. Pinto AGA, Jorge MSB. Prática clínica na estratégia saúde da família: relações entre equipe e usuários no território urbano. Rev Eletrônica Gest Saúde [Internet]. 2015 [citado em 18 jun 2017]; 6(2):1514-29. DOI: http://dx.doi.org/10.18673/gs.v6i2.22483

18. Campos CMS, Silva BRB, Forlin DC, Trapé CA, Lopes IO. Práticas emancipatórias de enfermeiros na Atenção Básica à Saúde: a visita domiciliar como instrumento de reconhecimento de necessidades de saúde. Rev Esc Enferm USP. [Internet]. 2014 [citado em 18 jun 2017]; 48(Esp):119-25. DOI: 10.1590/S0080-623420140000600017

19. Bezerra RS, Carvalho EL. Profissionais de saúde e dispositivos de mediação na atenção básica. In: Pinheiro R, Martins PH, editores. Usuários, redes sociais, mediações e integralidade em saúde. Rio de Janeiro: UERJ/IMS/LAPPIS; 2011. p. 149-60.

20. Gazzinelli MF, Souza V, Fonseca RMGS, Fernandes MM, Carneiro ACLL, Godinho LK. Práticas educativas grupais na atenção básica: padrões de interação entre profissionais, usuários e conhecimento. Rev Esc Enferm USP. [Internet] 2015 [citado em 18 jun 2017]; 49(2):284-91. DOI:http://dx.doi.org/10.1590/S0080623420150000200014

21. Campos GWS. Um método para análise e co-gestão de coletivos. São Paulo: Hucitec; 2000.

22. Cecílio LCO. As necessidades de saúde como conceito estruturante na luta pela integralidade e eqüidade na atenção em saúde. In: Pinheiro R, Mattos RA, editores. Os sentidos da integralidade na atenção e no cuidado à saúde. Rio de Janeiro: Cepesc; 2001. p. 113-126.

23. Sancho LG, Silva NEK. Descortinando o acesso aos serviços de saúde na perspectiva da interdisciplinaridade: debate de ideias. Physis [Internet]. 2013 [citado em 18 jun 
2017]; 23(2):371-91.

http://dx.doi.org/10.1590/S0103-

73312013000200004
DOI: CONTRIBUIÇÕES

Antonio Germane Alves Pinto contribuiu na concepção do objeto de estudo, na coleta e análise dos dados, redação e revisão. Maria Dayanne Luna Lucetti, Kelly Fernanda Silva Santana, Adriana de Moraes Bezerra e Maria Corina do Amaral Viana participaram na redação e revisão. Maria Salete Bessa Jorge atuou com o delineamento metodológico e análise dos dados.

\footnotetext{
Como citar este artigo (Vancouver)

Pinto AGA, Lucetti MDL, Santana FS, Bezerra AM, Viana MCA, Jorge MSB. Gestão do cuidado e da clínica no atendimento aos usuários da Estratégia Saúde da Família. REFACS [Internet]. 2019 [citado em inserir dia, mês e ano de acesso]; 7(1):21-29. Disponível em: inserir link de acesso. DOI: inserir link do DOI.

Como citar este artigo (ABNT)

PINTO, A. G. A. et al. Gestão do cuidado e da clínica no atendimento aos usuários da Estratégia Saúde da Família. REFACS, Uberaba, MG, v. 7, n. 1, p. 21-29, 2019. Disponível em: <inserir link de acesso>. Acesso em: inserir dia, mês e ano de acesso. DOI: inserir link do DOI.

Como citar este artigo (APA)

Pinto, A.G.A., Lucetti, M.D.L., Santana, F.S., Bezerra, A.M., Viana, M.C.A, \& Jorge, M.S.B. (2019). Gestão do cuidado e da clínica no atendimento aos usuários da Estratégia Saúde da Família. REFACS, 7(1), 21-29. Recuperado em: inserir dia, mês e ano de acesso de inserir link de acesso. DOI: inserir link do DOI.
} 